\title{
Pupillary Block
}

National Cancer Institute

\section{Source}

National Cancer Institute. Pupillary Block. NCI Thesaurus. Code C50716.

An obstruction of the pupil. 